\title{
Classification of Gravitational Instanton Symmetries
}

\author{
G. W. Gibbons and S. W. Hawking \\ Department of Applied Mathematics and Theoretical Physics, University of Cambridge, \\ Cambridge CB3 9EW, England
}

\begin{abstract}
We classify the action of one parameter isometry groups of Gravitational Instantons, complete non singular positive definite solutions of the Einstein equations with or without $\Lambda$ term. The fixed points of the action are of 2-types, isolated points which we call "nuts" and 2-surfaces which we call "bolts". We describe all known gravitational instantons and relate the numbers and types of the nuts and bolts occurring in them to their topological invariants. We perform a $3+1$ decomposition of the field equations with respect to orbits of the isometry group and exhibit a certain duality between "electric" and "magnetic" aspects of gravity. We also obtain a formula for the gravitational action of the instantons in terms of the areas of the bolts and certain nut charges and potentials that we define. This formula can be interpreted thermodynamically in several ways.
\end{abstract}

\section{Introduction}

There has been considerable interest recently in "Instantons" in Yang-Mills Theory [1-3]. They may be defined as non singular solutions of the classical equations in 4-dimensional Euclidean space. They provide stationary phase points in the path integral for the amplitude to tunnel between two topologically distinct vacua $[4,5]$ and they may play a role in confinement. Instantons also contribute to the anomalous divergence of the axial vector current [2] and they may lead to the decay of baryons into leptons. Because gravity and supergravity are gauge theories like Yang-Mills it seems reasonable to suppose that gravitational instantons may play a similar important role. We shall define a gravitational instanton to be a non singular complete positive definite metric which satisfies the classical vacuum Einstein equations or the Einstein equations with a $\Lambda$ term. The $\Lambda$ term can be regarded as a Lagrange multiplier for the 4-volume $V$ or it may arise from the Lagrangians of certain supergravity theories $[6,7]$. One class of gravitational instantons that has been extensively studied already is the KerrNewman family of metrics [7-12]. In these solutions one can remove the apparent 
singularity at the horizon and obtain a complete positive definite metric by identifying the imaginary time coordinate periodically. This leads to a new approach to the thermal aspects of black holes which have been discovered by other methods.

Nearly all known gravitational instantons possess continuous symmetry groups of at least two parameters [11, 14-19]. In this paper we shall give a classification scheme based on the existence of at least a one parameter group. This enables us to determine two kinds of basic object, one that we call a "nut" after the self-dual Taub-NUT solution $[10,11]$ which is the canonical example and the other which we call a "bolt", for obvious reasons. The canonical example is the Schwarzschild solution. In cases where the symmetry group is more than 1-dimensional, different choices of one-parameter subgroup may lead to different numbers and locations of nuts and bolts. However there are two topological invariants, the Euler number $\chi$ and the signature $\tau$, which can be expressed as sums over the nuts and bolts with certain coefficients. Roughly speaking, the Euler number is the sum of the number of nuts, the number of antinuts and twice the number of bolts while the signature is a measure of the number of nuts minus the number of antinuts.

The existence of these two kinds of basic objects reflects a certain symmetry in the theory analogous to duality invariance in electromagnetism. One can think of bolts as being the analogue of "electric" type mass-monopoles and the nuts as being gravitational dyons endowed with a real electric type mass-monopole and an imaginary "magnetic" type mass-monopole. The presence of magnetic type mass introduces a Dirac string-like singularity in the metric. This can be removed by appropriate identifications and changes in the topology of the spacetime manifold. However the metric cannot then be asymptotically flat in the usual sense. This means that the nuts unlike the bolts cannot occur in the classical regime. However one might imagine that quantum fluctuations of the metric might lead to the appearance and disappearance of nut-antinut pairs.

Gravitational Instantons can be interpreted as the stationary phase metrics in the path integrals for the partition functions, $Z$, of the thermal canonical ensemble [12] and the volume canonical ensemble [6]. In these cases the action of the instanton gives the dominant contribution to $-\log Z$. We shall relate this action to the areas of the bolts and to the charges and potentials of the nuts. From this it follows that the bolts have an intrinsic gravitational entropy equal to one quarter the sum of their areas. This generalises the results obtained for black holes and cosmological event horizons $[12,13,20]$.

\section{Nuts and Bolts}

We shall consider an oriented manifold $M$ with a positive definite metric $g_{a b}$ which admits at least a one-parameter isometry group $G$. We shall denote by $\mu_{\tau}: M \rightarrow M$ the action of the group, where $\tau$ is the group parameter and we shall denote by $K=K^{a} \frac{\partial}{\partial x^{a}}=\frac{\partial}{\partial \tau}$ the Killing vector. The isometry group $G$ is said to have a fixed point where $K=0$. At a fixed point $p$ the action of $\mu_{\tau}$ on the manifold $M$ gives rise to an isometry $\mu_{\tau} *: T_{p}(M) \rightarrow T_{p}(M)$ where $T_{p}(M)$ is the tangent space at $p . \mu_{\tau} *$ is 
generated by the antisymmetric matrix $K_{a ; b}$. Antisymmetric $4 \times 4$ matrices can have rank 0,2 , or 4 . The zero case is not interesting because it would imply that the Killing vector $K$ was zero everywhere and that the action of the group $G$ was trivial. This follows because $\mu_{\tau} *$ would be the identity and because $\mu$ commutes with the exponential map at $p$, i.e.

$$
\begin{aligned}
& \mu_{\tau} \circ \exp X=\exp \left(\mu_{\tau} *(X)\right) \\
& \forall X \in T_{p} .
\end{aligned}
$$

In the case that $K_{a ; b}$ has rank 2 there will be a 2-dimensional subspace $T_{1}$ of $T_{p}(M)$ which is left unchanged by $\mu_{\tau} *$. The action of $\mu_{\tau} *$ will rotate $T_{2}$, the 2-dimensional orthogonal complement of $T_{1}$ into itself. Thus $\mu_{\tau} *$ has the canonical form

$$
\mu_{\tau} *=\left(\begin{array}{cccc}
1 & 0 & 0 & 0 \\
0 & 1 & 0 & 0 \\
0 & 0 & \cos \kappa \tau & \sin \kappa \tau \\
0 & 0 & -\sin \kappa \tau & \cos \kappa \tau
\end{array}\right)
$$

where $\kappa$ is the surface gravity and is given by the non zero skew eigen value of $K_{a ; b}$ in an orthonormal frame. From this one can see that $\mu_{\tau} *$ and hence $\mu_{\tau}$ must be periodic with a period $2 \pi \kappa^{-1}$. The image of $T_{1}$ under the exponential map will not be moved by $\mu_{\tau}$ and so will constitute a 2-dimensional oriented totally geodesic sub manifold of fixed points. We shall call such a 2-dimensional fixed point set a bolt. A simple example is provided by the horizon 2-sphere of the Euclidean Schwarzschild solution with $G$ being the periodic group of imaginary time translations $[8,12]$.

In the case that $K_{a ; b}$ has the maximal rank 4 there can be no directions at $p$ which are left invariant under $\mu_{\tau} *$. Thus $p$ must be an isolated fixed point. We shall call it a nut after the fixed point at the centre of the Euclidean self-dual Taub-NUT solution [10]. In this case there will be two orthogonal 2-dimensional subspaces $T_{1}$ and $T_{2}$ which are mapped into themselves by $\mu_{\tau} *$. The canonical form is

$$
\mu_{\tau} *=\left(\begin{array}{cccc}
\cos \kappa_{1} \tau & \sin \kappa_{1} \tau & 0 & 0 \\
-\sin \kappa_{1} \kappa & \cos \kappa_{1} \tau & 0 & 0 \\
0 & 0 & \cos \kappa_{2} \tau & \sin \kappa_{2} \tau \\
0 & 0 & -\sin \kappa_{2} \tau & \cos \kappa_{2} \tau
\end{array}\right),
$$

where $\kappa_{1}$ and $\kappa_{2}$ are the skew eigenvalues of $K_{a ; b}$ in an orthonormal frame. For some purposes it is convenient to sub divide nuts into 2 classes - "nuts" and "antinuts" - depending on whether the sign of $\kappa_{1} \kappa_{2}$ is positive or negative respectively. Unless we explicitly say otherwise we shall call both classes nuts.

If $\kappa_{1} \kappa_{2}^{-1}=p q^{-1}$ where $p$ and $q$ are relatively prime integers, the action of $\mu_{\tau}$ will be periodic with period $\beta=2 \pi p \kappa_{1}^{-1}=2 \pi q \kappa_{2}^{-1}$. We shall call this a nut of type $(p, q)$.

If $\kappa_{1} \kappa_{2}^{-1}$ is irrational, the orbits of a vector $X$ in $T_{p}$ under the action of $\mu_{\tau} *$ is dense in the torus $C(X)$ consisting of all vectors $Y$ of the form

$$
\mu_{\tau_{1}}^{1} * \circ \mu_{\tau_{2}}^{2} *(X),
$$


where

$$
\begin{aligned}
& \mu_{\tau_{1}}^{1} *=\left(\begin{array}{cccc}
\cos \kappa_{1} \tau_{1} & \sin \kappa_{1} \tau_{1} & 0 & 0 \\
-\sin \kappa_{1} \tau_{1} & \cos \kappa_{1} \tau_{1} & 0 & 0 \\
0 & 0 & 1 & 0 \\
0 & 0 & 0 & 1
\end{array}\right), \\
& \mu_{\tau_{2} *}^{2}=\left(\begin{array}{ccccc}
1 & 0 & 0 & 0 & 0 \\
0 & 1 & 0 & \sin \kappa_{2} \tau_{2} \\
0 & 0 & \cos \kappa_{2} \tau_{2} & \cos \kappa_{2} \tau_{2}
\end{array}\right) .
\end{aligned}
$$

All scalar invariants of the metric must be constant over each torus in $M$ of the form $\exp C(X)$ for each $X \in T_{p}(M)$. Because scalar invariants characterize the metric it follows that $\mu_{\tau}^{1} *$ and $\mu_{\tau}^{2} *$ must actually correspond to independent isometries $\mu_{\tau}^{1}$ and $\mu_{\tau}^{2}$ of the metric $g_{a b}$ on $M$. One could then take appropriate combinations of the Killing vectors $K^{1}$ and $K^{2}$ such that the orbits were periodic. We shall therefore consider only periodic isometry groups.

The antisymmetric tensor $K_{a ; b}$ can be decomposed into self dual and antiself dual parts.

$$
K_{a ; b}=K_{a b}^{+}+K_{a b}^{-},
$$

where

$$
K_{a b}^{ \pm}=\frac{1}{2}\left(K_{a ; b} \pm \frac{1}{2} \varepsilon_{a b c d} K^{c ; d}\right) .
$$

At a bolt

$$
K_{a b}^{+} K^{+a b}=K_{a b}^{-} K^{-a b} .
$$

At a nut

$$
K_{a b}^{+} K^{+a b}>K_{a b}^{-} K^{-a b}
$$

while at an antinut

$$
K_{a b}^{+} K^{+a b}<K_{a b}^{-} K^{-a b} .
$$

A nut is said to be self-dual if $K_{a b}^{-}$is zero. Then $p=q= \pm 1$. If the curvature is selfdual - i.e. if

$$
R_{a b c d}=\frac{1}{2} \varepsilon_{a b e f} R_{c d}^{e f}
$$

then $K_{a ; b}$ is self-dual everywhere if it is self-dual at one point. Similar remarks apply to anti self-dual anti-nuts which have $p=-q= \pm 1$.

\section{Examples}

The examples of Schwarzschild and the self-dual Taub-NUT solutions have already been mentiod. The metric of the Schwarzschild solution can be written in the form

$$
d s^{2}=\left(1-2 M r^{-1}\right) d \tau^{2}+\left(1-2 M r^{-1}\right)^{-1} d r^{2}+r^{2}\left(d \theta^{2}+\sin ^{2} \theta d \phi^{2}\right) .
$$


The apparent singularity at the horizon $r=2 M$ can be removed by identifying $\tau$ with a period $8 \pi M[7,10,11]$. The radial coordinate then has the range $2 M \leqq r<\infty$ and the topology of the manifold is $R^{2} \times S^{2}$. The isometry group is $O(2) \otimes O(3)$ where the $O(2)$ corresponds to translations in the periodically identified imaginary time $\tau$ and the $O(3)$ corresponds to rotations of the $\theta$ and $\phi$ coordinates.

The Killing vector $\frac{\partial}{\partial \tau}$ has unit magnitude at large radius and has a bolt on the horizon $r=2 M$ which is a 2 -sphere of area $16 \pi M^{2}$. The surface gravity $\kappa=(4 M)^{-1}$ and the period $\beta$ is $8 \pi M$. A typical Killing vector of $O(3)$, for example $\frac{\partial}{\partial \phi}$, is zero on a non compact 2-surface, the axis, $0=0$ or $\pi$. We shall not consider Killing vector which have non compact bolts. One can also take linear combinations $p^{-1} 4 M \frac{\partial}{\partial \tau}+q^{-1} \frac{\partial}{\partial \phi}$. These will have a nut of type $(p, q)$ at the northpole $\theta=0$ of the horizon $r=2 M$ and an antinut of type $(p,-q)$ at the southpole, $\theta=\pi$, of the horizon $r=2 M$.

The Kerr solution with mass $M$ and (imaginary) angular momentum $=i \alpha M$ has a Euclidean section with metric

$$
\begin{aligned}
d s^{2}= & \left(r^{2}-\alpha^{2} \cos ^{2} \theta\right)\left(d r^{2} \Delta^{-1}+d \theta^{2}\right) \\
& +\left(r^{2}-\alpha^{2} \cos ^{2} \theta\right)^{-1}\left[\Delta\left(d \tau+\alpha \sin ^{2} \theta d \phi\right)^{2}+\sin ^{2} \theta\left(\left(r^{2}-\alpha^{2}\right) d \phi-\alpha d \tau\right)^{2}\right],
\end{aligned}
$$

where

$$
\Delta=r^{2}-2 M r-\alpha^{2} .
$$

The apparent singularity at the horizon

$$
r=r_{+}=M+\left(M^{2}+\alpha^{2}\right)^{1 / 2}
$$

can be removed by identifying the points $(r, \tau, \theta, \phi)$ with $(r, \tau+2 \pi \gamma, \theta, \phi+2 \pi \gamma \Omega)$ where

$$
\gamma=2 M r_{+}\left(M^{2}+\alpha^{2}\right)^{-1 / 2}
$$

and

$$
\Omega=\alpha\left(r_{+}^{2}-\alpha^{2}\right)^{-1}
$$

is the imaginary angular velocity $\frac{d \phi}{d \tau}$ of the horizon. This identification gives the manifold topology $R^{2} \times S^{2}$, the same as that of the Euclidean Schwarzschild solution. By the No Hair Theorems these are the only solutions which are asymptotically flat in the conventional sense.

The isometry group is $O(2) \otimes O(2)$ and is generated by $\frac{\partial}{\partial \phi}$ and the corotating Killing vector $K=\frac{\partial}{\partial \tau}+\Omega \frac{\partial}{\partial \phi}$. K is zero on the horizon which is the 2-sphere $r=r_{+}$with area $A=4 \pi\left(r_{+}^{2}-\alpha^{2}\right)$. The surface gravity $\kappa=\gamma^{-1}$ and the period $\beta=2 \pi \gamma$. The time translation Killing vector $\frac{\partial}{\partial \tau}$ has unit magnitude at large radius 
and for non zero $\alpha$ has 2 isolated fixed points at the north and south poles of the horizon. One of these is a nut and the other an anti-nut, which is being determined by the choice of orientation and the sign of $\alpha$. The surface gravities are

$$
\begin{aligned}
& \kappa_{1}=\gamma^{-1}, \\
& \kappa_{2}= \pm \Omega .
\end{aligned}
$$

If $M$ and $\alpha$ are such that $\gamma \Omega=q / p$, where $q$ and $p$ are relatively prime integers the nut and anti-nut will be of type $(p / q)$ and $(p,-q)$ respectively and the period will be $2 \pi p \gamma=2 \pi q \Omega^{-1}$.

The self-dual Taub-NUT metric can be written in the form

$$
\begin{aligned}
d s^{2}= & (r-n)(r+n)^{-1}(d \tau+2 n \cos \theta d \phi)^{2} \\
& +\left(r^{2}-n^{2}\right)\left(d \theta^{2}+\sin ^{2} \theta d \phi^{2}\right) \\
& +(r+n)(r-n)^{-1} d r^{2} .
\end{aligned}
$$

The Dirac string singularity at the northpole $(\theta=0)$ can be removed by introducing a new coordinate

$$
\tau^{\prime}=\tau+2 n \phi .
$$

Similarly the Dirac string singularity at the southpole $(\theta=\pi)$ can be removed by introducing a new coordinate

$$
\tau^{\prime \prime}=\tau-2 n \phi .
$$

Because $\phi$ is identified modulo $2 \pi$, $\tau^{\prime}$ and $\tau^{\prime \prime}$ must be identified modulo $8 \pi n$. These identifications and overlapping coordinate patches give the surfaces of constant $r>n$ the topology of 3 -spheres with $\left(\tau(2 n)^{-1}, \theta, \phi\right)$ being Euler angles. The apparent singularity at $r=n$ is in fact just the origin in hyperspherical polar coordinates. The topology of the manifold is $R^{4}$. The curvature is self-dual with the orientation defined by the positively oriented orthonormal basis

$$
\begin{aligned}
& \omega^{0}=(r+n)^{1 / 2}(r-n)^{-1 / 2} d r, \\
& \omega^{\prime}=\left(r^{2}-n^{2}\right)^{1 / 2}\left(\cos \left(\frac{\tau}{2 n}\right) d \theta+\sin \left(\frac{\tau}{2 n}\right) \sin \theta d \phi\right), \\
& \omega^{2}=\left(r^{2}-n^{2}\right)^{1 / 2}\left(-\sin \left(\frac{\tau}{2 n}\right) d \theta+\cos \left(\frac{\tau}{2 n}\right) \sin \theta d \phi\right), \\
& \omega^{3}=(r-n)^{1 / 2}(r+n)^{-1 / 2}(d \tau+2 n \cos \theta d \phi) .
\end{aligned}
$$

The isometry group is isomorphic to $U(2)=(U(1) \otimes S U(2)) / Z_{2}$. The $U(1)$ group is generated by the Killing vector $\frac{\partial}{\partial \tau}$ which is normalized to have unit magnitude at large $r$. It has a single self-dual nut fixed point at the origin $r=n$. The surface gravity $\kappa_{1}=\kappa_{2}=(4 n)^{-1}$ the period $\beta=8 \pi n$. The $S U(2)$ acts transitively on 3 -spheres of constant $r$. A typical Killing vector $\frac{\partial}{\partial \phi}$ also has a single fixed point at 
the origin $r=n$ but this time it is an anti-self dual anti-nut with $\kappa_{1}=-\kappa_{2}=1$ and period $2 \pi$.

Another metric of Taub-NUT form is [18]

$$
\begin{aligned}
d s^{2}= & \left(r^{2}-n^{2}\right)(r-2 n)^{-1}\left(r-\frac{1}{2} n\right)^{-1} d r^{2} \\
& +\left(r^{2}-n^{2}\right)\left(d \theta^{2}+\sin ^{2} \theta d \phi^{2}\right) \\
& +(r-2 r)\left(r-\frac{1}{2} n\right)\left(r^{2}-n^{2}\right)^{-1}(d \tau+2 n \cos \theta d \phi)^{2} .
\end{aligned}
$$

The curvature is not self-dual. The Dirac string singularities on the axis can be removed by identifying $\tau$ with period $8 \pi n$. As before this makes the surfaces of constant $r>2 n$ into 3 -spheres. The apparent singularity at $r=2 n$ corresponds to these 3 -spheres collapsing to a 2 -sphere. The isometry group is the same as for the self-dual Taub-NUT solution. The Killing vector $\frac{\partial}{\partial \tau}$ has unit magnitude at large $r$ and has a bolt of area $12 \pi n^{2}$ at $r=2 n$ with surface gravity $\kappa=(4 n)^{-1}$. The Killing vector $\frac{\partial}{\partial \phi}$ is a typical generator of the $S U(2)$ group. It has a nut of type $(1,2)$ at the north pole, $(\theta=0)$ and an antinut of type $(1,-2)$ at the southpole.

The multi-Taub-NUT metric [11] can be written in the form

$$
d s^{2}=V(d \tau+\omega \cdot d \mathbf{x})^{2}+V^{-1} d \mathbf{x} \cdot d \mathbf{x},
$$

where

$$
V^{-1}=1+\sum_{i} 2 n_{i}\left|\mathbf{x}-\mathbf{x}_{i}\right|^{-1}
$$

and

$$
\operatorname{curl} \omega=\operatorname{grad}\left(V^{-1}\right) .
$$

The $\mathbf{x}_{i}$ denote the distances in the 3-dimensional metric $d \mathbf{x} \cdot d \mathbf{x}$ the $i$ 'th nut with parameter $n_{i}$ and the grad and curl operations are also performed in this metric. The vector field $\omega(\mathbf{x})$ will have Dirac String singularities running from each nut. If the nut parameters $n_{i}$ are equal to a single value $n$ the singularities can all be removed by identifying $\tau$ with period $8 \pi n$. A large surface which surrounds all the nuts acquires the topology of the lens space $L(\mid, s)$ - a 3 -sphere with $s$ points identified, where $s$ is the number of nuts. Thus the metric is not asymptotically flat in the usual sense. The curvature is self-dual. In general for $s \geqq 3$ the only Killing vector will be $\frac{\partial}{\partial \tau}$. This has unit magnitude at large values of $r$ and a self-dual fixed point at each nut with $\kappa_{1}=\kappa_{2}=(4 n)^{-1}$.

Another family of self-dual metrics can be obtained from the multi-Taub-NUT form by omitting the constant term 1 in $V^{-1}$ in Eq. (3.18) [21]. Again to obtain a regular metric the $n_{i}$ have all to be equal but in this case they can be made equal to one by rescaling the coordinates. The topology and the nuts are the same as for the corresponding multi-Taub-NUT solutions, however unlike the multi-Taub-NUT case, these metrics are Asymptotically Locally Euclidean (A.L.E.). This means that they are asymptotic to Euclidean space identified under a discrete sub group of 
$S O(4)$. In fact they are the most general family of self-dual A.L.E. spaces with these identifications. Other self-dual A.L.E. instantons with different identifications have been found implicitly by Hitchin [22] but explicit metrics are not yet known.

When $s=1$ one obtains flat space. When $s=2$ one obtains the Eguchi-Hanson metric $[16,17]$. The metric can be written as

$$
\begin{aligned}
d s^{2}= & \left(1-a^{4} r^{-4}\right)^{-1} d r^{2}+\left(1-a^{4} r^{-4}\right) \frac{r^{2}}{4}(d \psi+\cos \theta d \phi)^{2} \\
& +\frac{r^{2}}{4}\left(d \theta^{2}+\sin ^{2} \theta d \phi^{2}\right) .
\end{aligned}
$$

The apparent singularity at $r=a$ can be removed by identifying $\psi$ modulo $2 \pi$ rather than modulo $4 \pi$ as is usual for Euler angles on $S^{3}$. The identification makes the surfaces of constant $r>a$ into $R P^{3}$, a 3 -sphere with antipodal points identified. At large values of $r$ the metric tends to that of flat Euclidean space, points reflected in the origin being identified. The surface $r=a$ is a 2-sphere.

The isometry group is $U(2)=(U(1) \times S U(2)) / Z_{2}$ the same as for Taub-NUT space of which this metric is a limiting form. The $U(1)$ subgroup is generated by the Killing vector $\frac{\partial}{\partial \psi}$ which has a bolt on the 2-sphere $r=a$ of area $\pi a^{2}$ with surface gravity $\kappa=1$ and period $2 \pi$. The Killing vector $\frac{\partial}{\partial \phi}$, a typical generator of the $S U(2)$ group, has 2 isolated fixed points, self dual nuts at the north and south poles of the 2-sphere $r=a$. These have $\kappa_{1}=\kappa_{2}$ and the period is $2 \pi$.

We now turn to complete non-singular solutions of the Einstein equations with positive $\Lambda$ term:

$$
R_{a b}=\Lambda g_{a b} ; \quad \Lambda>0 .
$$

These are all compact [23].

The simplest example is a 4 -sphere of radius $3^{1 / 2} \Lambda^{-1 / 2}$ in 5 -dimensional Euclidean space. This is the analytic continuation of de Sitter space [20]. The isometry group is the $S O(5)$ of rotations about the origin in 5-dimensional space. These are generated by $5 \times 5$ anti-symmetric matrices which can have rank 0,2 , or 4. The zero case is trivial. In the case of rank 2 there is a 3-plane through the origin of $R^{5}$ which is not moved by the rotation. The intersection of this with the 4-sphere is a bolt which is a 2 -sphere with area $12 \pi \Lambda^{-1}$. With the normalization of the Killing vector from $0(5)$, the period is $2 \pi$, the surface gravity is 1 . However for physical applications [20] it may be convenient to choose the Killing vector to have unit magnitude on the orbit which is a geodesic. In this case the period is $2 \pi 3^{1 / 2} \Lambda^{-1 / 2}$. The surface gravity is $(\Lambda / 3)^{1 / 2}$. If the matrix is of rank 4 , there will be one direction through the origin in $R^{5}$ which is left unchanged by the rotation. The intersection of this direction with the 4-sphere will constitute a nut and an antinut.

The next example is complex projective plane, $C P^{2}$, with its standard Kaehler metric $[14,15]$ which has an anti-self-dual Weyl tensor. This can be realized as $C^{3}-\{0\}$ with coordinates $Z_{1}, Z_{2}, Z_{3}$ factored by the equivalence relation 
$\left(Z_{1}, Z_{2}, Z_{3}\right) \equiv\left(\lambda Z_{1}, \lambda Z_{2}, \lambda Z_{3}\right), \lambda \in C-\{0\}$. The isometry group is $S U(3) / Z_{3}$ which acts on the coordinates in the standard manner. This is generated by traceless antiHermitean matrices. Such matrices can be divided into two classes, those with two equal eigenvalues and those with 3 unequal eigenvalues. In the case one gets an anti-self-dual antinut which can be taken to be at the "origin" $\left(0,0, Z_{3}\right)$ and a bolt at "infinity" which is the 2-sphere $\left(Z_{1}, Z_{2}, 0\right)$. In the latter case one gets 3 isolated fixed points two of which will be antinuts and one a nut. They can be located at the origin $\left(0,0, Z_{3}\right)$, the northpole of infinity $\left(Z_{1}, 0,0\right)$ and the southpole $\left(0, Z_{2}, 0\right)$.

One can describe these fixed points in more detail by introducing coordinates.

$$
\begin{aligned}
& Z_{1} / Z_{3}=r \cos \left(\frac{\theta}{2}\right) \exp i(\psi+\phi) / 2, \\
& Z_{2} / Z_{3}=r \sin \frac{\theta}{2} \exp i(\psi-\phi) / 2 .
\end{aligned}
$$

The metric then takes the form

$$
\begin{aligned}
d s^{2}= & d r^{2}\left(1+\frac{1}{6} \Lambda r^{2}\right)^{-2}+\frac{1}{4} r^{2}\left(1+\frac{1}{6} \Lambda r^{2}\right)^{-2}(d \psi+\cos \theta d \phi)^{2} \\
& +\frac{1}{4} r^{2}\left(1+\frac{1}{6} \Lambda r^{2}\right)^{-1}\left(d \theta^{2}+\sin ^{2} \theta d \phi^{2}\right) .
\end{aligned}
$$

The Killing vector $\frac{\partial}{\partial \psi}$ corresponds to a generator of $S U(3)$ with 2 equal eigenvalues. It has an antiself dual nut at $r=0$ with surface gravity $\kappa_{1}=-\kappa_{2}=\frac{1}{2}$ and period $4 \pi$. It has a bolt at $r=\infty$ with area $6 \pi \Lambda^{-1}$ and $\kappa=\frac{1}{2}$. The Killing vector $\frac{\partial}{\partial \phi}$ corresponds to a generator of $S U(3)$ with 3 unequal eigenvalues. It has a self-dual nut at the origin $r=0$ with $\kappa_{1}=\kappa_{2}=1$ and period $2 \pi$. It is an antinut of type $(1,-2)$ at the northpole of the sphere at infinity $r=\infty, \theta=0$ and an antinut of type $(1,-2)$ at the southpole $r=\infty, \theta=\pi$.

Our next example is the metric product of two 2-spheres each with radius $\Lambda^{-1 / 2}$ and area $4 \pi \Lambda^{-1}$. It can be regarded as a limiting case of the Schwarzschildde Sitter solution [15] with the surface gravities of the black hole and cosmological horizons equal. The isometry group is $S O(3) \otimes S O(3)$, the two factors acting on the 2-spheres independently. A circle subgroup of the full isometry group can be projected into circle subgroups in the two factors. If one of these projections consists of the identity only, the corresponding Killing vector has 2 bolts which are 2 -spheres of area $4 \pi \Lambda^{-1}$. In the other case there will be 4 isolated fixed points, two nuts and two antinuts.

The only other known gravitational instanton with positive $\Lambda$ is an $S^{2}$ bundle over $S^{2}$ discovered by Page [19] as a limiting case of the Kerr-de Sitter solution. The metric can be written in the Taub-NUT form:

$$
\begin{aligned}
d s^{2}= & \frac{3}{\Lambda}\left(1+v^{2}\right)\left\{\left(1-v^{2} \cos ^{2} \varrho\right)\left(3-v^{2}-v^{2}\left(1+v^{2}\right) \cos ^{2} \varrho\right)^{-1} d \varrho^{2}\right. \\
& +\left(1-v^{2} \cos ^{2} \varrho\right)\left(3+6 v^{2}-v^{4}\right)^{-1}\left(d \theta^{2}+\sin ^{2} \theta d \phi^{2}\right) \\
& +\left(3-v^{2}-v^{2}\left(1+v^{2}\right) \cos ^{2} \varrho\right)\left(3+v^{2}\right)^{-2}\left(1-v^{2} \cos ^{2} \varrho\right)^{-1} \sin ^{2} \varrho \\
& \left.\cdot \frac{1}{4}(d \psi+\cos \theta d \phi)^{2}\right\},
\end{aligned}
$$


where $v$ is the positive root of

$$
v^{4}+4 v^{3}-6 v^{2}+12 v-3=0
$$

which works out to be

$$
0.2817 \text {. }
$$

The surfaces of constant $\varrho, 0<\varrho<\pi$, are 3 -spheres on which $(\psi, \theta, \phi)$ are Euler angle coordinates. The apparent singularities at $\varrho=0$ and $\varrho=\pi$ are where the 3 -spheres collapse to 2 -spheres.

The isometry group is $U(2)=(U(1) \otimes S U(2)) / Z_{2}$, the same as for Taub-NUT. The Killing vector $\frac{\partial}{\partial \psi}$ generates the $U(1)$ subgroup and has bolts at the 2 -spheres $\varrho=0$ and $\varrho=\pi$ with areas

$$
12 \pi\left(1-v^{4}\right)\left(3+6 v^{2}-v^{4}\right)^{-1} \Lambda^{-1}
$$

The Killing vector $\frac{\partial}{\partial \phi}$, a typical generator of the $S U(2)$ subgroup, has 4 isolated fixed points at $0=0$ or $\pi$ and $\varrho=0$ or $\pi$. Two of these are nuts and two antinuts.

Compact gravitational instantons with negative or zero $\Lambda$ are known but they cannot admit any continuous isometry group. We shall therefore not consider them in this paper.

Non compact solutions with negative $\Lambda$ are also known. These may admit continuous isometrics but they do not seem to play a role in path integrals.

\section{Topological Invariants}

There are two topological invariants which can be expressed as integrals of the curvature of a 4-dimensional metric. For a compact manifold these are the Euler number

$$
\chi=\left(128 \pi^{2}\right)^{-1} \int_{M} \varepsilon_{a b}^{e f} R_{e f g h} \varepsilon_{c d}^{g h} R^{a b c d} \sqrt{g} d^{4} x
$$

and the signature (sometimes called the index)

$$
\tau=\left(96 \pi^{2}\right)^{-1} \int_{M} R_{a b c d} \varepsilon^{c d e f} R_{e f}^{a b} \sqrt{g} d^{4} x .
$$

For non compact manifolds there are additional boundary terms. For $\chi$ these are [24]

$$
\left(128 \pi^{2}\right)^{-1} \int_{\partial M}\left(R_{a b c d} K^{a c} n^{b} n^{d}+64 \operatorname{det} K_{b}^{a}\right) \sqrt{h} d^{3} x,
$$

where $n^{a}$ is the outward directed normal to the boundary $\partial M, K_{a b}=n_{c ; d} h_{a}^{c} h_{b}^{d}$ is the second fundamental form and $h_{a b}=g_{a b}-n_{a} n_{b}$ is the induced metric on $\partial M$. For $\tau$ the boundary terms are [25]

$$
-2\left(96 \pi^{2}\right)^{-1} \int R_{a b c d} \varepsilon^{c d e f} n^{a} n_{e} K_{f}^{b} \sqrt{h} d^{3} x-\eta(0),
$$

where $\eta(s)$ is a quantity constructed from the eigenvalues of a certain differential operator on the boundary $\partial M$. 
The Euler number in the alternating sum of the Betti numbers

$$
\chi[M]=\sum_{p=0}^{p=4}(-1)^{p} B_{p},
$$

where the $p$ 'th absolute Betti number, $B_{p}$, is the rank of the $p^{\prime}$ th absolute homology group $H_{p}(M)$. It is also the rank of the $4-p$ 'th relative cohomology group $H^{4-p}(M, \partial M)$. These cocycles may be represented by closed $4-p$ forms which vanish on the boundary $\partial M$ and which are not the exterior derivative of a $4-p-1$ form which itself vanishes on the boundary. For a compact manifold without boundary $B_{0}=B_{4}=1$ and $B_{1}=B_{3} . B_{1}=0$ if the manifold is simply connected as in all our examples. If there is a boundary $B_{0}=1$ and $B_{4}=0$. In a compact manifold without boundary $B_{p}$ is equal to the number of linearly independent harmonic $p$-forms.

In the case of a compact manifold without boundary $\tau$ may be defined as the signature of the quadratic form on $\mathrm{H}_{2}(M)$ given by the intersection number between two 2-cycles, i.e. the number of times they intersect, intersections being counted positive or negative according to whether or not the orientation of the tangent space of the intersection point agrees with the orientation arising from the tangent planes to the two 2-surfaces. In the case of the intersection number of a cycle with itself one slightly distorts one copy of a representative of the 2-cycle so that it intersects the other copy transversely. A necessary and sufficient condition that the manifold admit a spinor structure is that the self intersection numbers of the 2-cycles must be even for simply connected manifolds [26].

$\tau$ is also equal to the quadratic form on $H^{2}(M)$ defined by the cup product. If one represents two elements of $H^{2}(M)$ by closed 2-forms, then the cup product is just

$$
\int_{M} \alpha \wedge \beta
$$

From this it follows that $\tau$ is equal to the number of linearly independent self-dual harmonic 2-forms minus the number of antiself dual ones.

In the case of a manifold with boundary $\partial M$, the cup product may be defined between $H^{2}(M)$ and $H^{2}(M, \partial M)$ by the above formula, i.e. between closed 2-forms which are not the exterior derivatives of 1-forms and closed 2-forms which vanish on $\partial M$. With the natural injection $H^{2}(M, \partial M)$ into $H^{2}(M)$, this defines a quadratic form on $H^{2}(M, \partial M)$. The quadratic form may have zero eigenvalues but $\tau$ may be defined as the number of positive eigenvalues minus the number of negative eigenvalues [25]. The definition of $\tau$ by homology for manifolds with boundary is simply the dual of the above. One can define the intersection number between a cycle in the absolute homology group $H_{2}(M)$ and one in the relative homology group $\mathrm{H}_{2}(M, \partial M)$ i.e. the group of equivalence classes of 2-chains whose boundaries lie in $\partial M$ and which are not the boundaries of 3-chains. With natural injection of $\mathrm{H}_{2}(M)$ into $\mathrm{H}_{2}(M, \partial M)$ this defines a quadratic form on $\mathrm{H}_{2}(M)$. The quadratic form will have zero eigenvalues corresponding to elements of $\mathrm{H}_{2}(M)$ which are homologous to elements of $H_{2}(\partial M)$ but $\tau$ will be equal to the number of positive eigenvalues minus the number of negative eigenvalues. 
The values of $\chi$ and $\tau$ for the examples in the previous section are

$\begin{array}{lll}\text { Schwarzschild and Kerr } & \chi=2 & \tau=0 \\ \text { Self-dual Taub-NUT } & \chi=1 & \tau=0 \\ \text { Multi-Taub NUT } & \chi=s & \tau=s-1 \\ \text { Non self dual Taub-NUT } & \chi=2 & \tau=1 \\ \text { Eguchi-Hanson } & \chi=2 & \tau=1 \\ S^{4} & \chi=2 & \tau=0 \\ C P^{2} & \chi=3 & \tau=-1 \\ S^{2} \times S^{2} & \chi=4 & \tau=0 \\ \text { Twisted } S^{2} \times S^{2} & \chi=4 & \tau=0 .\end{array}$

The relevance of $\chi$ and $\tau$ to our nuts and bolts classification is given by various fixed point theorems which relate them both to the zeros of vector fields on the manifold. For isometries these theorems take particularly simple forms [27-30]. On a compact manifold the Euler number is given by

$$
\chi[M]=N_{+}+N_{-}+\sum_{i} \chi_{i}
$$

Where $N_{+}$and $N_{-}$are the number of nuts and antinuts and $\chi_{i}$ is the Euler number of the $i$ 'th bolt. In all our examples $\chi_{i}=2$. Indeed this will necessarily be the case if the manifold $M$ is simply connected and if the bolt can not be continuously deformed to a point (it can be so deformed in $S^{4}$ but not in the other examples). This is the analogue of the theorem that the horizon of a black hole metric with Lorentz signature $(-+++)$ must have an event horizon that is topologically spherical [31]. This formula also holds for manifolds with boundary $\partial M$ provided that the Killing vector field is either everywhere tangential to the boundary (as it is in all our examples) or is everywhere transverse. The theorem for signature is rather more complicated. On compact manifolds

$$
\tau=\sum_{\text {nuts }}-\operatorname{cotan} p \theta \operatorname{cotan} q \theta+\sum_{\text {bolts }} Y \operatorname{cosec}^{2} \theta,
$$

$Y$ is the self intersection number of a bolt and $2 \theta$ is the group parameter.

Equation (4.7) holds for all values of $\theta$. If one expands in powers of $\theta$ the first two terms give

$$
\begin{aligned}
& \sum_{\text {nuts }}-(p q)^{-1}+\sum_{\text {bolts }} Y=0, \\
& \sum_{\text {nuts }} \frac{1}{3}\left(p q^{-1}+q p^{-1}\right)+\frac{1}{3} \sum_{\text {bolts }} Y=\tau .
\end{aligned}
$$

Applying (4.8) to the Killing vector $\frac{\partial}{\partial \psi}$ in $C P^{2}$ which has an antinut with $-p=q=1$ and a bolt one finds that the self intersection number of the bolt must be -1 . This shows that $C P^{2}$ does not admit a spin structure though it can have a generalized spin structure [32-33].

In the case of a non compact there is an additional boundary term $\eta(\theta, 0)$ in Eq. (4.7). The quantity $\eta(\theta, s)$ is formed out of the eigenvalues of a certain differential operator on the boundary $\partial \boldsymbol{M}[25]$. 


\section{Duality}

The action of the group $G$ with Killing vector $K=\frac{\partial}{\partial \tau}$ defines a fibering $\pi: M$ $-C \rightarrow B$ where $C$ is the fixed point set of $\mu_{\tau}$. In other words $B$ is the 3-dimensional space of non-trivial orbits of $G$. The manifold $B$ inherits a metric

$$
h_{a b}=g_{a b}-V^{-1} K_{a} K_{b},
$$

where $V=K^{a} K_{a}$. The metric $g_{a b}$ on $M$ can then be written locally in the form

$$
d s^{2}=V\left(d \tau+\omega_{i} d x^{i}\right)^{2}+V^{-1} \gamma_{i j} d x^{i} d x^{j},
$$

where $\left\{x^{i}\right\}$ are coordinates on $B, \gamma_{i j}=V h_{i j}$ and $\omega_{i}$ are independent of the fourth coordinate $\tau$. The vector field $\omega_{i}$ in $B$ is defined up to a "gauge transformation"

$$
\tau^{\prime}=\tau+\lambda\left(x^{\prime}\right)
$$

under which

$$
\omega_{i}^{\prime}=\omega_{i}-\frac{\partial}{\partial x^{i}} \lambda .
$$

The twist field $H_{i j}=\partial_{i} \omega_{j}-\partial_{j} \omega_{i}$ is gauge invariant. It can be expressed as

$$
H_{a b}=2 K_{e ; f} h_{b}^{e} h_{a}^{f} V^{-1} \text {. }
$$

For the rest of this section we shall work in the 3-dimensional space $B$. Indices $i, j, k$ etc. will be raised or lowered by $\gamma_{i j}$ and covariant differentiation with respect to $\gamma_{i j}$ will be denoted by $\|$. Using the 3-dimensional alternating tensor one can define a twist vector $H_{i}$ by

$$
H_{i}=\frac{1}{2} \varepsilon_{i}^{j k} H_{j k} .
$$

This obeys the conservation equation

$$
H_{\| i}^{i}=0 \text {. }
$$

One can therefore define the nut charge within a 2-surface $L$ by

$$
N=(8 \pi)^{-1} \int_{L} H_{i} d \sigma^{i} .
$$

In the case of a nut of type $(p, q)$

$$
N=(8 \pi p q)^{-1} \beta .
$$

For a bolt with self intersection number $Y$

$$
N=(8 \pi)^{-1} Y \beta .
$$

These formulae are obtained by expanding the metric in a Taylor series in normal coordinates about the fixed point set. From them one can see that Eq. (4.8) is an expression of the fact that a compact manifold has zero total nut chare. 
One can project the field equations, $R_{a b}=\Lambda g_{a b}$, into $B$

$$
\begin{aligned}
V^{-1} R_{a b} K^{a} K^{b}= & -V \nabla^{i} \nabla_{i} \log V+\frac{1}{2} V^{3} H_{i} H^{i}, \\
R_{c d} h_{i}^{c} h_{j}^{d}= & { }^{3} R_{i j}-\frac{1}{2} V^{-2} V_{i} V_{j}+\gamma_{i j} \nabla_{k} \nabla^{k} \log V \\
& +\frac{1}{2} V^{2} H_{i} H_{j}-\frac{1}{2} V^{2} H_{k} H^{k} \gamma_{i j}, \\
V^{-1 / 2} R_{c d} h_{j}^{c} K^{d}= & \frac{1}{2} V^{-1} \varepsilon_{j}^{p q} \nabla_{p} H_{q},
\end{aligned}
$$

where ${ }^{3} R_{i j}$ is the Ricci tensor of the metric $\gamma_{i j}$.

Adding (5.11) and (5.12) one obtains an expression for the 4-dimensional action of the metric

$$
\begin{aligned}
\hat{I}= & -\frac{1}{16 \pi} \int_{M} \sqrt{g} d^{4} x(R-2 \Lambda)-\frac{1}{8 \pi} \int_{\partial M} K \sqrt{b} d^{3} x \\
= & -\frac{\beta}{16 \pi} \int_{B} \sqrt{\gamma} d^{3} x\left({ }^{3} R-2 \Lambda V^{-1}-\frac{1}{2} V^{-2} \nabla_{i} V \nabla^{i} V-\frac{1}{2} V^{2} H_{i} H^{i}\right) \\
& -\frac{\beta}{8 \pi} \int_{\partial B} k \sqrt{c} d^{2} x,
\end{aligned}
$$

where $b_{a b}$ is the induced metric and $K=K_{a}^{a}$ is the trace of the second fundamental form of $\partial M$ in the metric $g_{a b}$ and $c_{i j}$ is the induced metric and $k=k_{i}^{i}$ is the trace of the second fundamental form in the metric $\gamma_{i j}$. To obtain the field Eqs. (5.11)-(5.13) one requires that $\hat{I}$ be stationary under variations of $\gamma_{i j}, V$ and $H_{i}$ subject to the constraint (5.7). That expresses the fact that $H$ is the curl of $\omega_{i}$. One therefore defines a new quantity $\tilde{I}$ by adding the constraint multiplied by a Lagrange multiplier

$$
\begin{aligned}
\tilde{I}= & -\frac{\beta}{16 \pi} \int_{B} \sqrt{\gamma} d^{3} x\left({ }^{3} R-2 V^{-1} \Lambda-\frac{1}{2} V^{-2} \nabla_{i} V \nabla^{i} V-\frac{1}{2} V^{2} H_{i} H^{i}\right) \\
& -\frac{\beta}{8 \pi} \int_{B} k \sqrt{c} d^{3} x+\frac{1}{16 \pi} \beta \int_{B} \sqrt{\gamma} d^{3} x \psi \nabla^{i} H_{i} .
\end{aligned}
$$

Variation of $H_{i}$ gives the equation

$$
V^{2} H_{i}=\nabla_{i} \psi \text {. }
$$

We shall therefore call $\psi$ the nut potential. The fact that the nut potential exists is equivalent to the field equation $R_{a b} K^{a} h_{c}^{b}=0$. One then rewrites $\tilde{I}$ as

$$
\begin{aligned}
\tilde{I}= & \frac{1}{16 \pi} \beta \int_{B} \sqrt{\gamma} d^{3} x\left({ }^{3} R-2 V^{-1} \Lambda-\frac{1}{2} V^{-2}\left(\nabla_{i} V \nabla^{i} V-\nabla_{i} \psi \nabla^{i} \psi\right)\right) \\
& -\frac{1}{8 \pi} \beta \int_{\partial B} k \sqrt{c} d^{2} x+\frac{1}{16 \pi} \beta \int_{\partial B} \psi \psi^{i} V^{-2} d \sigma_{i} .
\end{aligned}
$$

Variation of $V$ and $\gamma_{i j}$ in (5.18) gives the field equations

$$
R_{a b} K^{a} K^{b}=\Lambda V \quad \text { and } \quad R_{a b} h_{c}^{a} h_{d}^{b}=\Lambda h_{c d} .
$$


Variation of $\psi$ in (5.18) gives the constraint

$$
\nabla^{i}\left(V^{-2} \psi_{i}\right)=0 .
$$

One can therefore regard (5.18) as the effective action for a 3-dimensional relativity theory with metric $\gamma_{i j}$ on $B$ and non-linear fields $\psi$ and $V$.

The term

$$
V^{-2}\left(\nabla_{i} V \nabla^{i} V-\nabla_{i} \psi \nabla^{i} \psi\right)
$$

in (5.18) is the Lagrangian of an $O(2,1)$ non-linear $\sigma$ model. That is $(5.20)$ can be regarded as the metric on 2-dimensional de Sitter space with coordinates $V$ and $\psi$. This metric has the 3-parameter group of isometries $S L(2 R)$ which can be realized as the following:

1. Translations $\quad \psi \rightarrow \psi+a$

$$
V \rightarrow V \text {; }
$$

2. Dilations $\quad \psi \rightarrow b \psi$

$$
V \rightarrow b V \text {; }
$$

3. The Ehlers Transform $[34,35]$

$$
\begin{aligned}
& \psi \rightarrow \frac{\psi+b\left(V^{2}-\psi^{2}\right)}{(1-b \psi)^{2}-b^{2} V^{2}}, \\
& V \rightarrow \frac{V}{(1-b \psi)^{2}-b^{2} V^{2}} .
\end{aligned}
$$

Corresponding to these 3 symmetries of (5.20) there will be three Noether currents

$$
\begin{aligned}
& J_{T}^{i}=-V^{-2} \nabla^{i} \psi, \\
& J_{D}^{i}=V^{-1} \nabla^{i} V-\psi V^{-2} \nabla^{i} \psi, \\
& J_{E}^{i}=2 \psi V^{-1} \nabla^{i} V-V^{-2}\left(\psi^{2}+V^{2}\right) \nabla^{i} \psi .
\end{aligned}
$$

If $\Lambda=0$, these are symmetries of the effective action $\tilde{I}$ and so the Noether currents are all conserved in the metric $\gamma_{i j}$

$$
J_{T \| i}^{i}=J_{D \| i}^{i}=J_{E \| i}^{i}=0 .
$$

If $\Lambda$ is non zero, it breaks the symmetry under the dilation and Ehler's transforms. Thus

$$
\begin{aligned}
& J_{T \| i}^{i}=0, \\
& J_{D \| i}^{i}=2 V^{-1} \Lambda, \\
& J_{E \| i}^{i}=2 \psi V^{-1} \Lambda .
\end{aligned}
$$

In the vacuum case the symmetry under the 3-parameter group expresses the duality between the electric aspects of gravity, characterized by $V$, and the magnetic or nut aspects characterized by $\psi$. A particularly simple case is when $V=\psi$. Then $\gamma_{i j}$ is the flat metric and one obtains the two families of multi TaubNUT metrics. 


\section{Action and Entropy}

In the case of a compact manifold $M$ integration of Eq. (5.32) gives the total action, $\hat{I}$

$$
\begin{aligned}
\hat{I} & =-\frac{1}{8 \pi} \int_{M} \Lambda \sqrt{g} d^{4} x \\
& =-\frac{1}{8 \pi} \beta \int_{B} \frac{\Lambda}{V} \sqrt{\gamma} d^{3} x \\
& =-\frac{1}{16 \pi} \beta \int_{\partial B} J_{D}^{i} d \sigma_{i} .
\end{aligned}
$$

The boundary $B$ will consist of a disjoint set of 2 spheres around each nut and each bolt. Thus

$$
\hat{I}=-\frac{1}{16 \pi} \beta \sum_{n} Q_{n},
$$

where the dialation charge of the $n$ 'th nut and bolt is

$$
Q_{n}=\int_{\partial B_{n}} J_{D}^{i} d \sigma_{i}
$$

The $Q_{n}$ 's are not invariant under the translation $\psi \rightarrow \psi+a$ but in the case of a bolt one can define an invariant quantity

$$
M_{n}=8 \pi Q_{n}-\psi_{n} N_{n},
$$

where $\psi_{n}$ is the value of the nut potential at the $n$ 'th bolt and $N_{n}$ is its nut charge. The quantity $M_{n}$ can be regarded as the "mass" of the $n$ 'th bolt. It can be represented as an integral of the 2 -form over the bolt

$$
M_{n}=\frac{1}{8 \pi} \int K^{c ; d} d \Sigma_{c d} .
$$

$M_{n}$ obeys the Smarr relation

$$
2 \beta M_{n}=A_{n} \text {. }
$$

$A_{n}$ is the area of the $n^{\prime}$ th bolt. Thus

$$
\begin{aligned}
\hat{I} & =\sum_{\text {bolts }}-\frac{1}{4} A_{n}-\sum_{\text {bolts }} \frac{1}{2} \psi_{n} N_{n} \beta+\sum_{\text {nuts }}-\frac{1}{2} \psi_{n} N_{n} \beta \\
& =\sum_{\text {bolts }}-\frac{1}{4} A_{n}-\sum_{\text {bolts }} \frac{\psi Y \beta^{2}}{16 \pi}-\sum_{\text {nuts }} \frac{\psi \beta^{2}}{16 \pi p q} .
\end{aligned}
$$

This generalizes the formula

$$
\hat{I}=-\frac{1}{4} A
$$

which was found for $S^{4}[12]$.

Equation (6.9) can be interpreted thermodynamically in at least two ways. In the first approach one regards the $\Lambda$ term as part of the dynamics of theory. One then defines the partition function $Z$ for the canonical ensemble.

$$
Z=\sum_{n}\left\langle g_{n} \mid g_{n}\right\rangle
$$

where $\left|g_{n}\right\rangle$ is an orthonormal basis of states for the gravitational field with the given value of $\Lambda$. In this case, unlike the normal thermal canonical ensemble, there 
is no externally imposed temperature or chemical potential. The partition function $Z$ simply counts the total number of states $\left|g_{n}\right\rangle$. Since each of these is equally probable, the probability $P_{n}=Z^{-1}$ and thus the entropy $S=-\Sigma P_{n} \log P_{n}=\log Z$. The partition function $Z$ can also be represented as a path integral over all metrics $g$ on a compact manifold $M$

$$
Z=\int d[g] \exp -\hat{I}[g] \text {. }
$$

By the stationary phase approximation one would expect the dominant contribution to come from metrics near a solution, $g_{0}$, of the classical Einstein field equations and the value of $Z$ to be given approximately by exp $-\hat{I}\left[g_{0}\right]$. Thus

$$
\begin{aligned}
S= & -\hat{I}\left[g_{0}\right] \\
= & \sum_{\text {bolts }} \frac{1}{4} A_{n}+\sum_{\text {bolts }} \frac{\psi_{n} Y \beta^{2}}{16 \pi} \\
& +\sum_{\text {nuts }} \frac{\psi \beta^{2}}{16 \pi p q} .
\end{aligned}
$$

This shows that not only do bolts have an entropy equal to $\frac{1}{4}$ of their area, as was found for de Sitter space $[12,20]$, but there is also a contribution from the NUT charges of both nuts and bolts. Because of the translational freedom $\psi \rightarrow \psi+a$, this latter contribution to the entropy cannot be attributed to individual nuts and bolts, but its total value is invariant, since the sum of the NUT charges is zero for a compact manifold.

One can also regard the $\Lambda$ term as not being part of the field equations but as Lagrange multiplier or chemical potential for the 4-volume $V$. In this case, one can form the partition function for the volume canonical ensemble as

$$
Z[\Lambda]=\sum_{n}\left\langle g_{n}\left|\exp -\frac{\Lambda V_{n}}{8 \pi}\right| g_{n}\right\rangle \text {. }
$$

As before, one can represent $Z[\Lambda]$ as a path integral over all metrics and by the stationary phase approximation one expects the dominant contribution to $\log Z$ to be $-\hat{I}\left[g_{0}\right]$ where $g_{0}$ is a solution with the given value of $\Lambda$. On dimensional grounds

$$
\hat{I}\left[g_{0}\right]=-\frac{\Lambda V_{0}}{8 \pi}=-\frac{f}{8 \pi \Lambda},
$$

where $f$ may depend on the topology of the manifold and on the particular class of solutions (if there is more than one) but it is independent of $\Lambda$. From $Z[\Lambda]$ one can calculate the expectation value of the volume in the ensemble

$$
\langle V\rangle=-8 \pi \frac{\partial}{\partial \Lambda} \log Z \text {. }
$$

With the stationary phase value for $\log Z$ one obtains

$$
\langle V\rangle=V_{0} \text {. }
$$

One can also form an "entropy" for the canonical ensemble

$$
S_{V}=-\sum P_{n} \log P_{n}
$$

where the probability of being in the $n$ 'th state, $P_{n}$, equals

$$
Z^{-1} \exp -\left(\frac{\Lambda V_{n}}{8 \pi}\right)
$$


Thus

$$
S_{V}=-\Lambda^{2} \frac{\partial}{\partial \Lambda}\left(\Lambda^{-1} \log Z\right)
$$

using the stationary phase value one obtains

$$
S_{V}=-2 \hat{I}\left[g_{0}\right] \text {. }
$$

This differs by a factor 2 from entropy in the canonical ensemble in which $\Lambda$ was regarded as part of the field equations.

A physical interpretation of $S_{V}$ can be obtained in the following way. Let $N(V) d V$ be the number of states of the gravitational field with volumes between $V$ and $V+d V$. Then $Z[\Lambda]$ can be regarded as the Laplace transform of $N(V)$

$$
Z[\Lambda]=\int_{0}^{\infty} N(V) \exp -\left(\frac{\Lambda V}{8 \pi}\right) d V .
$$

Thus $N(V)$ is the inverse Laplace transform

$$
N(V)=\frac{1}{16 \pi^{2} i} \int_{-i \infty}^{+i \infty} Z[\Lambda] \exp \left(\frac{\Lambda V}{8 \pi}\right) d \Lambda .
$$

The contour in Eq. (6.24) should be taken to pass to the right of the essential singularity at $\Lambda=0$. The dominant contribution to $N(V)$ will come from the stationary points of (6.24) which occurs at

$$
\Lambda=\Lambda_{\text {s }}
$$

for which

$$
\frac{V}{8 \pi}=-\frac{\partial \log Z}{\partial \Lambda}=\left\langle\frac{V}{8 \pi}\right\rangle .
$$

The value of the integrand at the stationary phase point is $\exp S_{V}$. Thus

$$
\log N(V) \approx S_{V}
$$

In the case of a compact manifold the dilation symmetry was broken by the presence of the $\Lambda$ term. In the non-compact case one is interested in situations where the metric is asymptotically flat, either in the 3 or 4 dimensional senses. Both these require that $\Lambda=0$. In the 4-dimensional case, the boundary conditions are dilation invariant. This means that the change of the action under a dilation is just given by the total dilation charge

$$
\begin{aligned}
\frac{\delta \hat{I}}{\delta b} & =\frac{\delta \tilde{I}}{\delta b} \\
& =\int J_{D}^{i} d \sigma_{i} \\
& =Q_{D} \\
& =0 .
\end{aligned}
$$

However, under a dilation $g_{a b} \rightarrow k^{2} g_{a b}, \hat{I}\left[k^{2} g_{a b}\right]=k^{2} \hat{I}\left[g_{a b}\right]$. This shows that the action $\hat{I}[g]$ must be zero for a solution of the field equations that is asymptotically flat in the 4-dimensional sense. The Positive Action Theorem [36-41] then implies 
that the metric is flat if it is asymptotically Euclidean i.e. it approaches the standard flat metric on $R^{4}$ outside some compact subset in which the topology may differ from that of $R^{4}$. If the metric is asymptotically locally Euclidean, i.e. outside of a compact subset it approaches the standard flat metric on $R^{4}$ identified under some discrete subgroup $S O(4)$ which acts freely, then the Generalized Positive Action Conjecture $[42,43]$ implies that metric must be self-dual or antiself-dual.

One is also interested in metrics which the "spatial" metric approaches a flat 3-dimensional metric and in which $V$ approaches some constant value which can be normalized to unity by an appropriate choice of $\beta$.

The boundary condition $V=1$ at infinity is not preserved under the dilation transformation. To impose this constraint one can define a new quantity

$$
I^{\prime}=\tilde{I}+\frac{\beta}{16 \pi} \int_{\infty} \mu V \sqrt{c} d^{2} x
$$

where $\mu$ is a Lagrange multiplier. If one requires $I^{\prime}$ to be stationary under variations of $V$ which do not vanish on the boundary one obtains

$$
\mu=-V_{i} n^{i} V^{-2} \text {. }
$$

Then

$$
\begin{aligned}
\frac{\delta \hat{I}}{\delta b} & =\frac{\delta I^{\prime}}{\delta b} \\
& =\frac{\delta \tilde{I}}{\delta b}+\frac{\beta}{16 \pi} \int \mu V \sqrt{c} d^{2} x \\
& =-\frac{\beta}{16 \pi} \int V_{i} n^{i} V^{-1} \sqrt{c} d^{2} x \\
& =\frac{1}{2} \beta M_{\infty},
\end{aligned}
$$

where

$$
M_{\infty}=-\frac{1}{8 \pi} \int_{\infty} V_{i} n^{i} V^{-1} \sqrt{c} d^{2} x
$$

Thus

$$
\hat{I}=\frac{1}{2} \beta M_{\infty} \text {. }
$$

This generalizes the results obtained in reference [12] and it can also be applied to spaces such as Taub-NUT which are not asymptotically flat in the usual sense because the boundary surface at infinity cannot be embedded (even locally) in flat space. For Taub-NUT it gives a value of $4 \pi N^{2}$ in agreement with an unpublished calculation by Lincoln Davis. As in reference [12] one obtains an entropy equal to $1 / 4$ the area of the event horizon for the Schwarzschild and Kerr solutions.

One can also integrate the divergence of Ehler's current over the manifold. For a compact manifold this gives :

$$
\int \psi \Lambda \sqrt{g} d^{4} x=\sum_{\text {bolts }} 8 \pi \psi_{n} M_{n}-\sum_{\substack{\text { nuts } \\+ \\ \text { bolts }}} 4 \pi \psi_{n}^{2} N_{n} .
$$

We have not found a physical application for this result. 
Acknowledgements. We should thank J. F. Adams, M. F. Atiyah, N. Hitchin, D. N. Page and C. N. Pope for help and discussions.

\section{References}

1. Polyakov, A.: Phys. Lett 59 B, 82 (1975)

2. 't Hooft, G.: Phys. Rev. Lett. 37, 8 (1976)

3. Belavin, A., Polyakov, A., Schwarz, A., Tyupkin, B.: Phys. Lett. 59 B, 85 (1975)

4. Jackiw, R., Rebbi, C.: Phys. Lett. 37, 172 (1976)

5. Callan, C., Dashen, R., Gross, D.: Phys. Lett. 63 B, 334 (1976)

6. Hawking, S.W.: Nucl. Phys. B 144, 349 (1978)

7. Zumino, B.: Ann. N.Y. Acad. Sci. 302, 545 (1977)

8. Hartle, J., Hawking, S.W.: Phys. Rev. D 13, 2188 (1976)

9. Gibbons, G.W., Perry, M.J.: Phys. Rev. Lett. 36, 985 (1976)

10. Gibbons, G.W., Perry, M.J.: Proc. R. Soc. A 358, 467 (1978)

11. Hawking, S.W.: Phys. Lett. 60 A, 81 (1977)

12. Gibbons, G.W., Hawking, S.W.: Phys. Rev. D 15, 2752 (1977)

13. Hawking, S.W.: Commun. Math. Phys. 43, 199 (1975)

14. Eguchi, T., Freund, P.G.O.: Phys. Rev. Lett. 37, 1251 (1976)

15. Gibbons, G.W., Pope, C.N.: Commun. Math. Phys. 61, 239 (1978)

16. Eguchi, T. Hanson, A.: Phys. Lett. 74 B, 249 (1978)

17. Belinski, V.A., Gibbons, G.W., Page, R.N., Pope, C.N.: Phys. Lett. 76 B, 433 (1978)

18. Page, D.N.: Phys. Lett. 78 B, 249 (1978)

19. Page, D.N. : Phys. Lett. 79 B, 235-238 (1978)

20. Gibbons, G.W., Hawking, S.W.: Phys. Rev. D 15, 2738 (1977)

21. Gibbons, G.W., Hawking, S.W.: Phys. Lett. 78 B, 430 (1978)

22. Hitchin, N.J.: Polygons and gravitons. Preprint, Oxford University. Math. Proc. Camb. Phil. Soc. (in press)

23. Milnor, J.: Morse theory. Princeton: Princeton University Press (1963)

24. Chern, S.S.: Ann. Math. 46, 674 (1945)

25. Atiyah, M.F., Patodi, V.K., Singer, I.M. : Proc. Cambridge Philos. Soc. 77, 43 (1975); 78, 405 (1975)

26. Geroch, R.P.: J. Math. Phys. 9, 1739 (1968)

27. Atiyah, M.F., Bott, R.: Ann. Math. 87, 451 (1968)

28. Bott, R.: Mich. Math. J. 14, 231-244 (1967)

29. Atiyah, M.F., Singer, I.M.: Ann. Math. 87, 546-604 (1968)

30. Baum, P., Cheeger, J.: Topology 8, 173-193 (1969)

31. Hawking, S.W.: Commun. Math. Phys. 25, 152-166 (1972)

32. Hawking, S.W., Pope, C.N.: Phys. Lett. 73 B, 42 (1978)

33. Back, A., Freund, P.G.O., Forger, M.: Phys. Lett. 77 B, 181 (1978)

34. Ehlers, J.: In: Les theories relativistes de la gravitation. Paris: CNRS 1959

35. Geroch, R.: J. Math. Phys. 13, 394 (1972)

36. Gibbons, G.W., Hawking, S.W., Perry, M.J.: Nucl. Phys. B 138, 141 (1978)

37. Page, D.N.: Phys. Rev. D 18, 2733-2738 (1978)

38. Gibbons, G.W., Pope, C.N.: Commun. Math. Phys. (in press)

39. Hawking, S.W.: Phys. Rev. D 18, 1747 (1978)

40. Gibbons, G.W., Hawking, S.W.: Proof of the positive action conjecture and the nature of the gravitational Action. Unpublished Report

41. Shoen, R.M., Yau, S.T.: Phys. Rev. Lett. 42, 547-548 (1979)

42. Hawking, S.W., Pope, C.N.: Nucl. Phys. B 146, 381-392 (1978)

43. Hawking, S.W.: Euclidean quantum gravity. Cargese Summer School Lectures 1978, New York, London: Plenum Press (in press)

Communicated by R. Geroch

Received December 19, 1978 